\title{
Cambios en el concepto aprendizaje de estudiantes de pedagogía: análisis de disponibilidad léxica y grafos ${ }^{*}$
}

\author{
Changes in the concept of learning in pedagogy students: \\ lexical availability and graphs analysis
}

\section{MARCO F. VALENZUELA CASTELLANOS ${ }^{1}$, M. VICTORIA PÉREZ VILLALOBOS $^{2}$, CLAUDIO BUSTOS ${ }^{3}$, PEDRO SALCEDO LAGOS ${ }^{4}$}

\author{
${ }^{1}$ Departamento de Psicología, Universidad de Concepción, Chile \\ Correo electrónico: marcovalenzuela@udec.cl \\ ${ }^{2}$ Departamento de Psicología, Universidad de Concepción \\ Correo electrónico: marperez@udec.cl \\ ${ }^{3}$ Departamento de Psicología, Universidad de Concepción \\ Correo electrónico: clbustos@udec.cl \\ ${ }^{4}$ Departamento de Psicología, Universidad de Concepción \\ Departamento Metodología de la Investigación e Informática Educativa, \\ Universidad de Concepción \\ Correo electrónico: psalcedo@udec.cl
}

La presente investigación utiliza análisis de disponibilidad léxica y grafos léxicos para analizar el cambio de concepciones de aprendizaje en futuros profesores. Se aplicaron pruebas de disponibilidad léxica al inicio y al término de un semestre a 97 estudiantes de Pedagogía. Se realizó análisis de disponibilidad léxica, así como la construcción de dos grafos léxicos, uno por cada observación. Los resultados muestran el reemplazo del $50 \%$ de las palabras mencionadas entre ambas observaciones, mientras que los grafos léxicos muestran características estructurales similares a aquellos construidos con grandes bases de datos. Los cambios observados en la disponibilidad léxica y en la conectividad de las palabras muestran el paso de un concepto de aprendizaje fundado en la enseñanza, a uno basado en procesos internos del aprendiz. Se discuten las implicancias del uso de estas metodologías para el estudio del lenguaje y el cambio conceptual.

Palabras clave: aprendizaje, cambio conceptual, grafos, disponibilidad léxica, profesores en formación

\footnotetext{
Esta Investigación se enmarca en el Proyecto FONDECYT 1120694, denominado Impacto de un programa de docencia para facilitar la autorregulación del aprendizaje mediante TIC.
} 
This research uses lexical availability and lexical graphs to analyze changes on the conceptualization of learning in future teachers. Lexical availability tests were applied to 97 pedagogy students. Lexical availability analysis was performed on both assessment, and two lexical graphs were constructed. Outcomes show the replacement of $50 \%$ of the words mentioned between the two observations, while lexical graphs show similar structural characteristics to those built with large databases. Changes observed in lexical availability and words connectivity show the passage of a concept of learning based on teaching, to one based on internal processes of the learner. The implications of using these methodologies for the study of language and observed conceptual change are discussed.

Key words: learning, conceptual change, graphs, lexical availability, teaching students

\section{INTRODUCCIÓN}

Diversos autores han planteado que los procesos de formación docente debieran tener como objetivo el cambio de concepciones de aprendizaje y epistemologías personales de los futuros profesores hacia posturas constructivistas, como una manera de mejorar tanto los propios aprendizajes como sus estrategias de enseñanza futuras (Gómez, Guerra y González 2011; Hargreaves 2010; Windschitl 2002). Se espera, entonces, que los futuros docentes modifiquen sus concepciones de aprendizaje, desde aquellas ingenuas adquiridas de forma implícita en sus procesos formativos previos hacia otras más sofisticadas, producto del conocimiento teórico y disciplinar.

Una forma de estudiar este cambio en la forma de entender y conceptualizar el aprendizaje es a través de metodologías que permitan analizar cambios en lenguaje; específicamente, en las palabras o unidades léxicas utilizadas para conceptualizar el aprendizaje de los profesores en formación, y en la relación que se presenta entre éstas. Se puede entender el lenguaje como un sistema o red de términos interdependientes, en que el significado de cada uno resulta únicamente de la presencia simultánea de los otros (Collins y Loftus 1975; Neuman 2006; Saussure 2007). El significado asignado a las palabras puede variar de acuerdo al contexto de interacción específico (Wittgenstein 1999) y de forma más permanente debido a los procesos de aprendizaje (Solé et al. 2010).

La disponibilidad léxica (DL) es la línea de investigación que estudia el léxico disponible, entendido éste como el "caudal léxico utilizable en una situación comunicativa" (López 1996: 245) alojado en el lexicón mental de cada individuo, un almacén de palabras situado en la memoria semántica (Elman 2004). El estudio de la DL ha permitido conocer aspectos cuantitativos y cualitativos en las relaciones entre las palabras y la comprensión de conceptos (Salcedo y del Valle 2013).

Para este trabajo se consideró que la disponibilidad léxica permite el acceso al léxico latente de los participantes en torno al concepto de aprendizaje, un acervo de significados del cual no se está plenamente consciente y que emerge ante la presentación de una palabra 
estímulo (Ferreira et al. 2014). Sin embargo, para el estudio de los procesos de cambio de conceptos, los indicadores tradicionales de disponibilidad léxica sólo proveen información sobre las modificaciones de disponibilidad de las unidades léxicas, pero no entregan información sobre cómo cambian las relaciones entre éstas.

En el estudio de los procesos psicológicos y cognitivos relacionados con el aprendizaje y el desarrollo del lenguaje, la consideración de éste como una red dinámica de componentes interconectados ha estimulado la utilización de la teoría de grafos como instrumento de representación y medida (Borge-Holthoefer y Arenas 2010a; Steyvers y Tenenbaum 2005). Un grafo de lenguaje o red léxica puede construirse considerando a las palabras o a los participantes como unidad de análisis, permitiendo observar aspectos lingüísticos, como la estructura de un lenguaje en particular (Ferrer i Cancho y Solé 2001), o psicológicos y cognitivos, como los significados compartidos en una comunidad (Alexandridis y Maru 2012) o los procesos de recuperación de una palabra (Morais, Olsson y Schooler 2013). De este modo, el estudio del lenguaje que incorpora los grafos a los métodos de la disponibilidad léxica, permite representar y analizar procesos de cambio y reestructuración del conocimiento, ya sea en aspectos macro, como la estructura del lenguaje, y en niveles meso y micro, como pueden ser determinados conceptos y palabras, respectivamente.

En el presente artículo se utiliza un método basado en grafos de disponibilidad léxica para analizar cambios en las unidades léxicas y sus relaciones en referencia al concepto de aprendizaje en 97 estudiantes de pedagogía. Los objetivos específicos de este trabajo son: (1) describir cambios en la disponibilidad léxica respecto del concepto aprendizaje en dos observaciones; (2) describir la estructura de grafos construidos a partir de pruebas de disponibilidad léxica, y (3) describir cambios en el concepto aprendizaje en el lapso de un semestre lectivo en estudiantes de Pedagogía.

El artículo se organiza en las siguientes secciones: en la sección 2 se abordan los fundamentos teóricos relacionados a las concepciones de aprendizaje, disponibilidad léxica y teoría de grafos; en la sección 3 se presenta el método del estudio, y en la sección 4, los resultados cuantitativos y cualitativos del análisis de disponibilidad léxica y grafos. Finalmente, en la sección 5 se presenta la discusión de resultados y conclusiones.

\section{Fundamentación teórica}

\subsection{Concepciones de aprendizaje}

Las concepciones de aprendizaje de los estudiantes corresponden a las formas no siempre conscientes de entender el aprendizaje, desarrolladas a través de su experiencia vital y enseñanza formal (Sánchez 2005). Tales concepciones influirían en los propios procesos de aprendizaje como estudiantes, así como en la forma de afrontar la enseñanza como profesores (Pozo, Scheuer, Mateos y Pérez 2006). 
Desde la década de 1970 diversas investigaciones fenomenográficas han indagado las concepciones de aprendizaje de los estudiantes en diversos niveles de su formación. Los resultados de estas investigaciones (Marton y Säljö 2005; Paakkari, Tynjälä y Kannas 2011; Purdie et al. 1996; Rosário et al. 2006) han mostrado que existen dos dimensiones claramente diferenciadas: por una parte, una concepción de aprendizaje cuantitativa/ reproductiva o incremental, caracterizada por la utilización de procesos básicos de memorización, recuperación y aplicación del conocimiento, y por otra, una dimensión cualitativa en que el aprendizaje es visto como procesos de abstracción, comprensión y resignificación, implicando también el desarrollo y transformación personal del que aprende y la construcción social de significados (ver Tabla 1).

Tabla 1. Categorías de concepciones y teorías implícitas del aprendizaje y epistemologías subyacentes

\begin{tabular}{|c|c|c|c|c|c|c|}
\hline \multirow{2}{*}{\begin{tabular}{|c}
$\begin{array}{c}\text { Concepciones } \\
\text { de aprendizaje }\end{array}$ \\
Marton \& \\
Säljö (2005)
\end{tabular}} & \multicolumn{3}{|c|}{ Cuantitativo } & \multicolumn{3}{|c|}{ Cualitativo } \\
\hline & $\begin{array}{l}\text { Aumento } \\
\text { cuantitativo }\end{array}$ & Memorizar & $\begin{array}{l}\text { Adquisición y } \\
\text { utilización }\end{array}$ & $\begin{array}{l}\text { Abstracción } \\
\text { de significados }\end{array}$ & $\begin{array}{l}\text { Proceso inter- } \\
\text { pretativo }\end{array}$ & $\begin{array}{l}\text { Desarrollo como per- } \\
\text { sona }\end{array}$ \\
\hline $\begin{array}{l}\text { Purdie et al. } \\
\text { (1996) }\end{array}$ & $\begin{array}{l}\text { Aumentar } \\
\text { conocimiento }\end{array} \mid$ & $\begin{array}{l}\text { Memorizar y } \\
\text { reproducir }\end{array}$ & $\begin{array}{l}\text { Un medio } \\
\text { para un fin }\end{array}$ & Comprender & $\begin{array}{l}\text { Ver algo de } \\
\text { otra manera }\end{array}$ & Desarrollo personal \\
\hline $\begin{array}{l}\text { Rosário et al. } \\
\text { (2006) }\end{array}$ & $\begin{array}{l}\text { Incrementar } \\
\text { conocimientos }\end{array}$ & Memorizar & Aplicar & Comprender & $\begin{array}{l}\text { Ver las cosas } \\
\text { de otra ma- } \\
\text { nera }\end{array}$ & $\begin{array}{l}\text { Cambiar como persona, } \\
\text { realizarse }\end{array}$ \\
\hline $\begin{array}{l}\text { Paakkari et al. } \\
\text { (2011) }\end{array}$ & \multicolumn{2}{|c|}{$\begin{array}{l}\text { Reproducción de conoci- } \\
\text { miento adquirido }\end{array}$} & $\begin{array}{l}\text { Aplicación de } \\
\text { conocimiento }\end{array}$ & & $\begin{array}{l}\text { Desarrollo de } \\
\text { significados } \\
\text { personales }\end{array}$ & $\begin{array}{l}\text { Transformación del } \\
\text { pensamiento } \\
\text { Crecimiento personal } \\
\text { Construcción colectiva } \\
\text { de significado }\end{array}$ \\
\hline $\begin{array}{l}\text { Teorías } \\
\text { implícitas }\end{array}$ & \multicolumn{2}{|c|}{ Directa } & \multicolumn{3}{|c|}{ Interpretativa } & Constructiva \\
\hline $\begin{array}{l}\text { Pozo et al. } \\
\quad(2006)\end{array}$ & \multicolumn{2}{|c|}{$\begin{array}{l}\text { Determinación externa } \\
\text { Inmediato y aislado } \\
\text { Incremento de conocimien- } \\
\text { to y procedimientos como } \\
\text { piezas aisladas } \\
\text { Conocimiento como reflejo } \\
\text { de la realidad }\end{array}$} & \multicolumn{3}{|c|}{$\begin{array}{l}\text { Determinación externa (enseńanza), sobre un } \\
\text { aprendiz activo: descubre, recuerda, relaciona } \\
\text { a través de procesos cognitivos básicos que fa- } \\
\text { cilitan o dificultan el aprendizaje } \\
\text { Proceso que integra distintas fases temporales } \\
\text { El hacer y la práctica reiterada son importantes } \\
\text { Incremento de y complejidad del conoci- } \\
\text { miento. } \\
\text { Conocimiento como reflejo más o menos } \\
\text { ajustado a la realidad }\end{array}$} & $\begin{array}{l}\text { Determinación interna } \\
\text { Proceso de interacción } \\
\text { que resulta en reestruc- } \\
\text { turación, } \\
\text { reconstrucción, } \\
\text { transformación y } \\
\text { re-elaboración. } \\
\text { Conocimiento como } \\
\text { construcción del sujeto }\end{array}$ \\
\hline
\end{tabular}




\begin{tabular}{|c|l|l|l|}
\hline $\begin{array}{c}\text { Epistemología } \\
\text { subyacente }\end{array}$ & \multicolumn{1}{|c|}{ Dualismo/multiplicismo } & \multicolumn{2}{|c|}{ Relativismo } \\
\hline $\begin{array}{c}\text { Entwistle } \\
\text { \& Peterson } \\
\mathbf{( 2 0 0 4 )}\end{array}$ & $\begin{array}{l}\text { Conocimiento absoluto, provisto por una } \\
\text { autoridad } \\
\text { Múltiples perspectivas, opiniones de igual } \\
\text { valor } \\
\text { Conciencia del conocimiento como provi- } \\
\text { entre alternativas } \\
\text { sional }\end{array}$ & & $\begin{array}{l}\text { Compromiso con una } \\
\text { perspectiva personal ra- } \\
\text { zonada }\end{array}$ \\
\hline
\end{tabular}

Nota: adaptado de Martínez-Fernández (2007) y complementado por los autores de esta investigación.

También se ha observado la influencia de los procesos formativos en las concepciones de aprendizaje de futuros profesores. Tynjälä (1997) realizó una investigación con estudiantes finlandeses para analizar el cambio de concepciones de aprendizaje al participar en un curso de Psicología educacional con enfoque constructivista. Los resultados mostraron siete concepciones, algunas de ellas influidas por los contenidos y procesos de aprendizaje intencionados en el curso y que consideran al aprendizaje como: 1) producto de la determinación externa en línea con las teorías conductuales, ya sea por condicionamiento, observación o modelado; 2) como un proceso inevitable ligado al desarrollo, en línea con el pensamiento de Piaget; 3) como resultado del rol activo del aprendiz y sus disposiciones, reconociendo procesos intencionales y no intencionales, actividades de estudio y procesos cognitivos complejos como la metacognición y el pensamiento crítico; 4) desarrollo de estrategias, estilos y enfoques superficiales o profundos que responden a contextos y dominios particulares; 5) procesamiento de información, procesos de memoria y funcionamiento del cerebro, teorías de esquemas y estructuras de conocimiento y procesos de reestructuración; 6) proceso interpretativo a partir de la interacción con otros, vinculándose a veces con procesamiento de la información, y 8) un proceso creativo, que se alinea con perspectivas constructivistas de construcción y reestructuración de conocimientos previos.

En estudiantes de pedagogía y profesores chilenos, las concepciones de aprendizaje se han estudiado principalmente bajo el marco teórico de las Teorías implícitas del aprendizaje y la enseñanza (Pozo, Scheuer, Mateos y Pérez 2006). Estas teorías corresponderían a un conocimiento puesto en marcha según las condiciones de contexto, siendo claves en la resolución de problemas y en explicación que dan las personas de los eventos que ocurren en el proceso de enseñanza aprendizaje (Pozo 1993).

Estas teorías implícitas pueden alinearse con los tres grandes enfoques teóricos del aprendizaje desde la psicología: una Teoría Directa, alineada con las teorías conductuales y una epistemología dualista (i. e. determinantes externos); una Teoría Interpretativa, en línea con la teoría del procesamiento de información y también bajo una epistemología dualista; y, finalmente una Teoría Constructiva alineada con los enfoques de Piaget y Vygotsky y una epistemología más bien relativista (i. e. determinantes internos e interacciones con el objeto de conocimiento y con otros). 
Utilizando cuestionarios de dilemas se ha encontrado que estudiantes de pedagogía y profesores presentan perfiles mixtos, en los cuales, si bien predomina una teoría implícita, pueden aparecer elementos de las otras. Por ejemplo, Gómez y Guerra (2012) encontraron, en estudiantes de pedagogía que, aunque más del $50 \%$ de las respuestas a los dilemas correspondía a una teoría constructiva, sus respuestas restantes se repartían entre las teorías directa e interpretativa. Resultados similares se han encontrado en estudios transeccionales y longitudinales, permitiendo caracterizar perfiles en donde predomina una teoría interpretativa con elementos de teoría directa o de teoría constructiva (Cárcamo y Castro 2015; Gómez, Guerra y González 2010). Una mayor presencia del enfoque contructivista se puede observar también en los participantes de cursos avanzados (Cárcamo y Castro 2015) o luego de participar de un curso destinado a desarrollar una perspectiva constructivista (Gómez et al. 2010).

\subsection{Disponibilidad léxica}

Desde sus inicios en Francia, a mediados de la década de 1950 (López Morales 1995; Paredes 2012), los estudios de $D L$ se han basado en dos supuestos: 1) la inclusión de palabras o expresiones, consideradas centros de interés, relacionados con campos semánticos o situaciones de la vida cotidiana y 2) las palabras que un hablante menciona o escribe ante la presentación de tales centros de interés como estímulo en un tiempo determinado (Jiménez Catalán 2017). Considerando que las respuestas de los participantes en las pruebas de $D L$ pueden consistir en expresiones formadas por una o más palabras, estas son consideradas unidades léxicas (UL) (Dimitrijević 1969), mientras que cada una de las palabras diferentes es considerada como vocablo (Gallego 2014).

Existen diversas métricas que permiten estudiar la disponibilidad léxica, tanto a niveles individual y grupal para un contexto determinado, como a nivel de unidades léxicas, formadas por una o más palabras (Fernández-Merino 2011). A nivel individual, la medida principal es el promedio de respuestas por participantes $(X R)$. A nivel grupal, se encuentran el total de palabras diferentes o vocablos $(P D)$ y el Índice de Cohesión $(I C)$. El análisis de unidades léxicas individuales se realiza a través del Índice de Disponibilidad Léxica $(I D L)$, obtenido de la frecuencia y de la posición relativa con que aparece una unidad léxica, permitiendo conocer cuál es su accesibilidad y posible importancia (Paredes 2012).

\subsubsection{Investigaciones recientes en Disponibilidad léxica}

La búsqueda de artículos de revistas científicas que incluyen el término Disponibilidad léxica en el título y referidas al español como primer idioma, en bases WOS y Scopus y Google académico, arroja un total de 28 publicaciones entre los años 1993 y 2017. Las investigaciones revisadas pueden dividirse en dos grandes categorías: a) estudios de léxico general, que consideran todos o algunos de los 16 centros de interés tradicionales del Proyecto panhispánico de disponibilidad léxica (Paredes 2014), y b) estudios de léxico específico, con 
centros de interés diferentes a los tradicionales referidos a algún léxico particular, como léxico matemático, insultos, representaciones de la sexualidad, anglicismos, nuevas tecnologías, etc.

Trece de los artículos de disponibilidad léxica referidos al español como primera lengua son de léxico general. Los estudios del léxico general utilizan todos o parte de los 16 centros de interés propuestos por los primeros estudios realizados en Francia a mediados de la década de 1950, adoptados por el Proyecto panhispánico de disponibilidad léxica. Los centros de interés son: Partes del cuerpo, La ropa, Partes de la casa (sin muebles), Los muebles de la casa, Alimentos y bebidas, Objetos colocados en la mesa para la comida, La cocina y sus utensilios, La escuela: muebles y materiales, Calefacción, iluminación y medios de airear un edificio, La ciudad, El campo, Medios de transporte, Trabajos del campo y del jardin, Animales, Juegos y distracciones, Profesiones. Como se puede observar, los centros de interés refieren a objetos y situaciones concretas, más que conceptos abstractos (Paredes 2014).

El único estudio longitudinal de léxico general encontrado en esta revisión se realizó en cohortes entre el año 1990 y 2008 de población dominicana (Alba 2013) y utilizó los 16 centros de interés mencionados. Dos estudios indagaron el léxico disponible en poblaciones bilingües: Blas y Casanova (2001) estudiaron el léxico disponible en español en estudiantes preuniversitarios espańoles con bilingüismo castellano-valenciano, mientras que Santos (2015) estudió a estudiantes mexicanos de educación primaria con bilingüismo náayeri (cora) - espańol, adaptando los centros de interés a la cultura náayeri; por ejemplo, se agregan los centros de interés objetos sagrados, lo que hay en el pueblo, el cuamil y lo que hay en el monte.

Tres estudios relacionaron el léxico disponible con otras variables: HernándezMuñoz, Izura y Ellis (2006) utilizaron los 16 centros de interés, agregando el concepto abstracto Inteligencia, para correlacionar el léxico disponible con factores cognitivos en españoles; Hernández-Muñoz (2015) evaluó la competencia léxica adulta usando la disponibilidad léxica y la especialización académica en universitarios espańoles, utilizando 6 centros de interés. Cepeda et al. (2016) relacionaron la disponibilidad léxica con la comprensión lectora en estudiantes chilenos de 14 a 18 ańos, incluyendo los colores a 5 centros de interés tradicionales.

Cuatro estudios agregaron centros de interés adicionales. Garzón Segura y Penagos López (2016) incorporaron saludos y despedidas, temas de conversación, los colores, los deportes y la higiene y el cuidado personal en su estudio en estudiantes colombianos de primer semestre de universidad. Sánchez y Murillo (1993) y Murillo (1999) agregaron medios de comunicación en la investigación realizada en preescolares y escolares costarricenses. Rodríguez muñoz y Muñoz Hernández (2011) incorporaron los colores y la mar para indagar la disponibilidad del léxico de registro coloquial, extranjerismos, formas dialecto-patrimoniales, marcas, siglas y abreviaturas en estudiantes secundarios de la región de Almería, España. Finalmente, Cepeda, Granada y Pomes (2014) analizaron el léxico disponible en escolares de 6 y 7 años chilenos, agregaron el centro de interés los colores.

Cuatro estudios incorporaron otros marcos teóricos y metodológicos al estudio de la disponibilidad léxica. Tres estudios utilizaron la teoría de los prototipos para analizar el léxico de población española mayor a 20 años (Ávila-Muñoz 2016, 2017; Ávila-Muñoz y 
Sánchez-Sáez 2014). Henríquez, Mahecha y Mateus (2016) ocuparon el centro de interés el cuerpo humano para construir redes semánticas utilizando el software Dispografo con estudiantes colombianos de $5^{\circ}$ y $11^{\circ}$ grados.

La investigación sobre léxicos específicos es relativamente reciente; 13 de los 14 artículos encontrados pertenecen al periodo 2013-2017. Solamente uno es de fecha anterior, referido a anglicismos en el habla culta de españoles adultos con estudios superiores (González 2003). También referido a anglicismos, se ha indagado el léxico informático de estudiantes españoles de Traducción e interpretación (Luján y Bolaños 2014).

Dos artículos han investigado el léxico informático. Uno de estos estudios se realizó en México con jóvenes de 12 a 18 años y adultos con hijos de esas edades con los centros de interés: 1) Los muebles de la casa, aparatos electrónicos y electrodomésticos, 2) Entretenimiento, juegos y diversiones, 3) Internet y tecnologías de la información y comunicación, 4) Palabras que se abrevian en telefonía e Internet, 5) Emoticones (Rodríguez, López y Padilla 2014). Un segundo estudio se realizó usando los centros de interés informática y nuevas tecnologías en estudiantes universitarios españoles de primer curso, de entre 18 y 22 años, y adultosmayores jubilados, de entre 65 y 72 años (Tomé 2016).

Otra línea de investigación ha indagado modismos y cultura popular: en estudiantes mexicanos de tres niveles de educación formal (primaria, secundaria y universitaria) se estudiaron las groserías (Pérez 2015); en estudiantes secundarios del noreste argentino se investigó sobre mitos populares, la ciudad, el campo, insultos y diversiones nocturnas (Wingeyer 2014a, 2014b).

En México y Chile se ha investigado el léxico matemático. En México, Pacheco, Ponce-Castañeda y Palomares-Sánchez (2016) estudiaron los centros de interés función, gráfica, serie, ecuación, espacio y matriz, en estudiantes de Ciencias e Ingeniería. En Chile, en la Universidad de Concepción, la investigación se ha enfocado en estudiantes secundarios con los centros de interés los números, el álgebra, la geometría, y probabilidades, datos y azar (Salcedo y del Valle 2013; Ferreira, Salcedo y del Valle 2014) y en universitarios estudiantes de Pedagogía en matemática se agregó a los centros de interés antes nombrados, los de cálculo, estadística y estructura (del Valle, Salcedo y Ferreira 2016; Cerda, Salcedo, Pérez y Marín 2017). El trabajo de del Valle et al. (2016) se utilizó metodología de grafos para representar redes léxicas. Con estudiantes de la Facultad de Ciencias Físicas y Matemáticas se indagó el léxico disponible de los centros cálculo, algebra, estadistica, fisica, ecuación y geometría (Urzúa et al. 2006).

El campo semántico de la Política (politica, democracia, participación politica, gobierno y derechos) fue analizado en estudiantes salvadoreños de último curso de bachillerato de entre 15 y 19 años de edad (Bermúdez Valle, Bustillo Romero y Escobar Blanco 2016).

También se ha explorado temáticas como las representaciones sobre la sexualidad a partir de los centros de interés partes del cuerpo, profesiones y oficios, juegos y distracciones y ¿cómo insultamos? en estudiantes argentinos de los primeros y últimos cursos de educación secundaria (Colombo, Trevisán y Wingeyer 2013). Por otra parte, se exploraron los atributos de profesionalismo en estudiantes de medicina y médicos chilenos, utilizando el método de 
disponibilidad léxica para responder la pregunta ‘Cuáles son los atributos que debe tener un buen médico?' (Fasce et al. 2009).

Respecto de cambios en el léxico disponible debidos a procesos de enseñanza y aprendizaje las investigaciones con diseńo transeccional de Ferreira et al. (2014) y Salcedo y del Valle (2013), han mostrado resultados diversos según centro de interés y cohortes. Se observó que, en general, los participantes incrementaban su promedio de respuestas; sin embargo, no se observó diferencias en el número de vocablos en las comparaciones entre niveles y se presentan leves aumentos en los índices de cohesión en tres centros de interés (algebra, geometría y probabilidades y azar) y mantención en uno (números).

Otros estudios han observado las relaciones entre el léxico disponible y variables como la inteligencia (Dimitrejević 1969); el tipo de instrucción de un segundo idioma (Jiménez-Catalán y Alba Ojeda 2009a) y el sexo (Jiménez-Catalán y Ojeda Alba 2009b), entre otras.

\subsection{Redes asociativas y de disponibilidad léxica}

Las palabras conforman las unidades básicas del léxico que, según su disponibilidad o accesibilidad en la memoria, son traídas a la mano de acuerdo al contexto, ya sea éste de comunicación oral o escrita (Ferreira, Salcedo y del Valle 2014). La accesibilidad de estas palabras será mayor, en la medida que sus relaciones de conectividad sean también mayores (Ferrer i Cancho y Solé 2001).

A partir de la premisa de que existe una estructura subyacente en el léxico que permite conocer el significado de una palabra según aquellas con las cuales se relaciona, se ha propuesto el análisis de la disponibilidad léxica a través de grafos (Echeverría et al. 2008; Karelovic, 2010). Un grafo es un artefacto matemático, consistente en una red compuesta por nodos y aristas que los conectan (Borge-Holthoefer y Arenas 2010a).

Los grafos léxicos son redes asociativas que modelan diversas relaciones entre las palabras, ya sea de sinonimia, hiper o hiponimia, causa-efecto, instrumentales o conceptuales entre otras. Para la construcción de estas redes se considera que dos nodos o unidades léxicas están unidos por una arista si la unidad léxica $i$ evoca la unidad léxica $j$ en dos o más participantes (Borge-Holthoefer y Arenas 2010a).

Considerando estos aspectos, puede decirse que el uso de metodología de grafos, unida a la de disponibilidad léxica, permite observar no solo la accesibilidad o saliencia en la memoria de unidades léxicas, sino también la manera en que estas se estructuran conformando campos semánticos y sus variaciones en el tiempo, como producto de procesos de aprendizaje.

Usando grafos léxicos se ha estudiado el léxico disponible de inglés en hablantes nativos y estudiantes avanzados del idioma (Ferreira y Echeverría 2010), y en áreas específicas como la matemática (Ferreira et al. 2014) y la geometría (Salcedo y del Valle 2013).

Las principales métricas de los grafos son: (a) el grado, $k$, de un nodo que indica la cantidad de conexiones que tiene con otros nodos, (b) la distancia, $l$, que corresponde 
a la cantidad de aristas que separan a dos nodos cualesquiera en la red, (c) la distancia promedio, $L$, o promedio de las longitudes de camino más cortas entre todos los pares de nodos en una red y se encuentra estrechamente relacionada con (d) el diámetro de la red, $D$, que hace referencia a la máxima distancia entre dos nodos cualesquiera de la red (Steyvers y Tenenbaum 2005), y (e) la densidad del grafo que corresponde al coeficiente entre la cantidad de aristas existentes en grafo, versus las que podrían existir.

Dos medidas probabilísticas entregan información acerca de la estructura de conectividad de un grafo, estas son: (a) el coeficiente de agrupamiento, $C$, referido a la probabilidad de una mayor conectividad entre nodos de información similares, ya sea porque comparten características fonológicas o porque muestran asociación semántica y (b) la distribución de grado, $P(k)$, que alude a la probabilidad que un nodo específico esté conectado a un número dado de otros nodos (Steyvers y Tenenbaum 2005).

En cuanto a su topología o estructura, los grafos léxicos presentan características propias de redes complejas (Steyvers y Tenenbaum 2005), como estructuras de mundo pequeño (Watts y Strogatz 1998) y distribuciones de grado libres de escala (Barabási y Albert 1999).

Una estructura de mundo pequeño corresponde a una red con longitudes de camino promedio cortas $(L \approx 2-3)$ y un coeficiente de agrupamiento relativamente alto en comparación a un grafo aleatorio igualmente denso (Steyvers y Tenenbaum 2005). Un grafo aleatorio es un modelo teórico en el cual las aristas entre los nodos se conectan al azar siguiendo una distribución de Poisson; en estos grafos, el grado de un nodo es independiente del grado de sus nodos vecinos, situación que no se presenta en las redes de lenguaje (Newman, Strogatz y Watts 2001; Peñaranda-Ortega, Quiñones-Vidal y OscaLluch 2009).

En el caso de los grafos léxicos, la estructura de mundo pequeño se produciría porque existen unidades léxicas que, teniendo significados diferentes, actúan como puentes conectando regiones que de otro modo se encontrarían separadas por muchos vínculos (Motter et al. 2002). Las distancias cortas entre las unidades léxicas representarían la posibilidad de acceso rápido de éstas, ya sea en procesos voluntarios de recuperación como la navegación mental en búsqueda de un concepto específico, o asociaciones libres como la búsqueda de palabras que comiencen con una letra determinada (Ferrer i Cancho y Solé 2001).

Una red libre de escala es tipo de red que se caracteriza por presentar pocos nodos fuertemente conectados y muchos escasamente conectados (Steyvers y Tenenbaum 2005). En estas redes los nodos más conectados actúan como atractores de los que ingresan en la red, siguiendo el principio de agregación preferencial (Albert y Barabási 2002). Asimismo, el ingreso de nuevos nodos generaría nuevas conexiones entre los ya existentes (Dogorovtsev y Mendes 2001). En el caso de las redes léxicas, su crecimiento se produciría a través de la aparición de nuevas conexiones entre unidades ya existentes, así como por la incorporación de nuevas unidades, que se vincularían a aquellas unidades más accesibles y frecuentemente usadas (Steyvers y Tenenbaum 2005). 
La distribución libre de escala no es una característica de todas las redes de lenguaje, aunque sí la estructura de mundo pequeño. Redes construidas a partir de tareas de asociación libre muestran distribuciones de grado exponenciales (Karelovic 2010; Zortea et al. 2014), o ley de potencia con corte exponencial (Morais et al. 2013). Estas formas de distribución de grado presentan una mayor probabilidad de conectividad en sus nodos, indicando que cada nuevo nodo que ingresa, posee la misma posibilidad de ser enlazado que el resto (Vitevich 2008).

\section{Metodología}

Se empleó un diseño pre-experimental, con medición pre y post sin grupo control (Campbell y Stanley 1995). Se determinó este diseño por tratarse de una población específica de estudiantes, que además en diversas asignaturas deben tratar con contenidos que consideran teorías del aprendizaje o principios conductistas, cognitivos o constructivistas que son por lo demás palabras corrientes en el léxico de profesores, dificultando la disponibilidad de un grupo equivalente que no esté expuesto a los mismos contenidos.

\subsection{Participantes}

Participaron del estudio 97 alumnos de pedagogía de la Universidad de Concepción, 49 hombres (50.5\%) y 48 mujeres (49.5\%) con edades entre 18 y 31 años $(M=21.1 ; D T$ = 2.27), pertenecientes a cuatro especialidades: Ciencias Naturales, Historia y Geografía, Educación Musical y Educación General Básica. Los estudiantes asistían a cursos obligatorios de asignaturas que tenían entre sus contenidos la unidad de teorías de aprendizaje. La participación en el estudio fue voluntaria, previo consentimiento informado.

\subsection{Contexto de aplicación}

Los cursos tuvieron duración de un semestre (marzo a julio y agosto a diciembre) revisándose en ellos conceptos, teorías y aspectos epistemológicos del aprendizaje, con especial énfasis en la consideración del aprendizaje como un proceso activo de construcción de significados desde las perspectivas de Piaget y Vygotsky; incluyendo procesos y conceptualizaciones propias de éstas (Castorina y Baquero 2005; Rosas y Sebastián 2008); así como de los procesos y variables intra y extrapersonales implicadas en el aprendizaje escolar (Pintrich 2006; Pozo 1998).

Las actividades de las clases consideraron reflexión individual, en duplas y discusiones grupales y la contextualización y contraste de los contenidos del curso con la propia experiencia, así como investigación bibliográfica, observación de situaciones de aprendizaje en contextos reales y de fenómenos perceptuales que los estudiantes debían explicar según contenidos de revisión bibliográfica y de las clases realizadas. 


\subsection{Método de recolección de datos}

Se aplicó una Prueba de disponibilidad léxica en dos ocasiones: al inicio de un curso de Psicología Educativa y al finalizar el curso. Este instrumento consta de dos partes, en una primera parte se solicitan los datos sociodemográficos de los participantes (edad, sexo, carrera de proveniencia, año de ingreso y semestre de estudios); la segunda parte corresponde a la prueba propiamente tal. En ésta, se presentan por escrito las instrucciones ("Dispone de 2 (dos) minutos para escribir la mayor cantidad de palabras - frases que pueda o sepa-respecto del concepto que indique el instructor. Una vez finalizado el tiempo deje de escribir y espere una nueva instrucción"), seguidas de dos columnas en blanco y numeradas para que los participantes escriban las palabras que libremente recuerden asociadas al centro de interés, el Aprendizaje en nuestro caso, que el aplicador del instrumento expresa en voz alta.

\subsection{Procedimiento de análisis de la disponibilidad léxica}

Las listas de respuestas fueron estandarizadas para identificar las unidades léxicas a analizar. Para ello, se corrigieron todas las faltas de ortografía y se unificaron distintas respuestas en un mismo concepto, utilizando un diccionario y algoritmos especialmente construidos para su corrección. En particular, los verbos se consideraron en infinitivo, los plurales en singular, la expresión Zona de desarrollo próximo se consideró como $z d p$ y en caso de respuestas compuestas por más de una palabra, se consideraron por separado cuando había mención de ellas en secuencia en otros casos (por ejemplo, proceso cognitivo se separó en proceso y cognitivo).

Para el análisis de la Disponibilidad léxica se consideraron: el índice de disponibilidad léxica $(I D L)$, el promedio de respuestas $(X R)$, el número de unidades léxicas diferentes por cada centro (PD), y el índice de cohesión (IC) (Echeverría et al. 2008).

$\mathrm{El} I D L$, que puede alcanzar valores entre 0 y 1 , para cada palabra se calculó con la adaptación de la fórmula de Echeverría, Herrera, Moreno y Pradenas (1987) realizada por Salcedo, Ferreira, del Valle, Cerda y Friz (2014), donde $r$ es el máximo dentro del conjunto del de respuestas dadas por los participantes; 1 es un factor de ajuste de la posición de una palabra fijado convencionalmente en $0.9, \mathrm{y} f_{i}$ es el número de veces que se repite la palabra $x$ en la posición $i$.

El $I C$ se calcula dividiendo el promedio de respuestas $X R$ por el número de palabras diferentes PD (Paredes 2012).

$$
I D L=\sum_{i=1}^{r} \lambda^{(i-1)} \frac{f_{i}}{N}
$$




\subsection{Procedimiento de análisis de los grafos}

Se construyeron grafos asociativos orientados a la unidad léxica, con nodos que representan las diferentes unidades léxicas enunciadas por cada participante, con aristas representando la presencia simultánea de dos unidades léxicas en la respuesta de un participante. El peso de cada arista se determinó por el número de participantes que nombraron tanto la palabra $i$, como la palabra $j$ en sus respuestas. Se utilizó poda 2, lo que implica que sólo fueron consideradas aquellas unidades léxicas mencionadas por al menos dos de los participantes.

Para la construcción de los grafos se utilizaron el software R y la librería iGraph. Para su representación gráfica se utilizó el software de código abierto Gephi 0.8.2-beta (Gephi.org 2014).

Para el análisis de los grafos se calcularon (a) grado $k$ de cada nodo, (b) grado medio $<k>$ del grafo, (c) diámetro $D$ del grafo con pesos, (d) longitud media de camino, (e) distribución de grado, $P_{k}$, (f) coeficiente de agrupamiento global o transitividad, (g) coeficiente medio de agrupamiento, (h) coeficiente de mundo pequeño $\sigma$, (i) medida de mundo pequeńo w; (j) coeficiente de agrupamiento global o transitividad, y (k) coeficiente medio de agrupamiento. Las fórmulas se presentan en anexo al final del artículo.

\subsection{Procedimiento de análisis cualitativo}

Para observar el cambio en las concepciones se efectuó un análisis cualitativo seleccionando las unidades léxicas más conectadas de cada red. El procedimiento de selección de las unidades léxicas consistió en determinar un punto desde el cual cada red comenzaba a perder sus características estructurales, específicamente la longitud camino más corto promedio. Este procedimiento entregó un número variable de unidades léxicas para cada red.

Posteriormente, siguiendo las categorías presentes en la bibliografía revisada respecto de las concepciones de aprendizaje (Marton y Säljö 2005; Paakkari et al. 2011; Purdie et al. 1996; Rosário et al. 2006; Tynjälä 1997) y las teorías implícitas del aprendizaje (Pozo et al., 2006) presentes en la Tabla 1, se determinaron dos dimensiones, una correspondiente a procesos y resultados de tipo cuantitativo o incremental y cualitativo o de reestructuración, y otra a determinación externa e interna (locus).

Las unidades léxicas se presentaron a tres especialistas, más el autor de este estudio quienes las clasificaron según las dimensiones proceso/resultado y locus. Los índices kappa promedio mostraron una convergencia considerable para proceso/resultado $(\kappa=.74)$ y moderada para locus $(\kappa=.44)$. Las palabras en que no hubo acuerdo fueron sometidas a discusión y contraste con la teoría.

Para desambiguar el significado de algunas unidades léxicas, se analizaron las redes de vecinos, es decir con qué otras palabras se encontraban relacionadas. 


\section{Resultados}

\subsection{Análisis de disponibilidad léxica}

Los indicadores globales de disponibilidad léxica, muestran aumento en el total de respuestas y en el promedio de éstas por cada participante, disminución del total de unidades léxicas diferentes, y un leve incremento en el índice de cohesión (tabla 2). Entre las dos observaciones, se mantienen 192 unidades léxicas, desapareciendo un total de 203 e ingresando 191 unidades léxicas nuevas.

La comparación de los $I D L$ de las unidades léxicas entre las dos observaciones (tabla 3) muestra desaparición, incorporación y diferencias en la disponibilidad de unidades léxicas entre una y otra medida. En particular se aprecia aumento de disponibilidad léxica e incorporación de unidades léxicas correspondientes a contenidos y conceptos teóricos tratados en el curso como procesos y variables involucrados en el aprendizaje.

Tabla 2. Descriptivos generales de Disponibilidad léxica

\begin{tabular}{|l|c|c|}
\hline & Observación 1 & Observación 2 \\
\hline$N$ & 97 & 97 \\
\hline Total de respuestas & 1109 & 1383 \\
\hline$P D$ & 395 & 383 \\
\hline$X R$ & 11.43 & 14.26 \\
\hline$I C$ & 0.028 & 0.037 \\
\hline UL diferentes que permanecen & $192(48.6 \%)$ & \\
\hline UL diferentes que desaparecen & $203(51.4 \%)$ & \\
\hline UL nuevas & & $191(49.9 \%)$ \\
\hline
\end{tabular}

En ambas observaciones la unidad léxica conocimiento muestra la mayor disponibilidad, aumentando en el segundo momento. Sin embargo, las unidades léxicas que le siguen son diferentes; en la primera observación es seguida por enseñanza, profesor, alumno y proceso, mientras que, en la segunda, es seguida por proceso, cambio, motivación y constructivismo.

Por otra parte, las unidades léxicas que disminuyen se refieren en su mayoría a condicionantes externos del aprendizaje (por ejemplo, enseñanza, profesor, escuela, educación, enseñar) o a procesos poco definidos que según contexto podrían corresponder a diferentes tipos de procesos y resultados (memoria, conocer, estudiar). Junto al incremento en disponibilidad de conocimiento y proceso en la segunda observación, también aumentan 
cambio, motivación y constructivismo, entre las más disponibles, mientras que profesor y enseñanza, aunque disminuyen su disponibilidad se mantienen entre ellas.

Tabla 3. Unidades léxicas (UL) con mayor IDL en dos medidas

\begin{tabular}{|c|l|c|l|l|}
\hline & Unidad léxica & $I D L_{1}$ & Unidad léxica & $I D L_{2}$ \\
\hline 1. & Conocimiento & 0.339 & conocimiento & 0.415 \\
\hline 2. & Enseńanza & 0.330 & proceso & 0.264 \\
\hline 3. & Profesor & 0.226 & cambio & 0.217 \\
\hline 4. & Alumno & 0.199 & motivación & 0.193 \\
\hline 5. & Proceso & 0.130 & constructivismo & 0.192 \\
\hline 6. & Educación & 0.119 & conducta & 0.185 \\
\hline 7. & Escuela & 0.108 & enseñanza & 0.159 \\
\hline 8. & Aprender & 0.103 & profesor & 0.154 \\
\hline 9. & Memoria & 0.102 & metacognición / adquisición & 0.141 \\
\hline 10. & Conocer & 0.098 & construcción(+) & 0.135 \\
\hline 11. & Saber & 0.097 & aprender & 0.131 \\
\hline 12. & Enseńar & 0.085 & cognición & 0.125 \\
\hline 13. & Experiencia & 0.079 & aprendiz / saber & 0.120 \\
\hline 14. & Estudiar & 0.075 & experiencia & 0.112 \\
\hline 15. & leer(-) & 0.074 & piaget & 0.110 \\
\hline
\end{tabular}

Nota: (-) Unidades léxicas que desaparecen; (+) Unidades léxicas que se incorporan.

Dada la mayor disponibilidad de unidades léxicas relacionadas con determinantes externos y contextuales para el aprendizaje, así como de procesos poco definidos en la primera observación, podría decirse que predomina una concepción reproductiva y centrada en la enseńanza, mientras que en la segunda observación aparecen elementos más bien procesuales y centrados en el aprendiz, sugiriendo que luego de un semestre el concepto aprendizaje sería definido de otra manera por los estudiantes.

\subsection{Análisis de grafos léxicos}

Para la construcción de los grafos se consideraron solo aquellas unidades léxicas que fueron mencionadas por al menos dos participantes, de acuerdo al principio de que una conceptualización es un significado compartido (Harispe et al. 2013). Se observan 155 
unidades léxicas diferentes en la primera observación y 165 en la segunda. De estas, 96 unidades permanecen entre las dos observaciones, 59 desaparecen y 69 se incorporan como nuevas en la segunda red.

$\mathrm{Al}$ analizar las métricas de los grafos de las observaciones 1 y 2 (ver Tabla 4), se observa aumentos en cantidad de unidades léxicas (nodos) y aristas y grado medio $<k>$ del grafo indicando que, junto con aumentar el número de unidades léxicas compartidas por los participantes, también se forman nuevas relaciones entre ellas.

Las longitudes medias de camino en ambas observaciones son muy cortas, dando señales de estructuras de mundo pequeño (Watts y Strogatz 1998), confirmadas por el coeficiente de mundo pequeño, $\sigma$; y la medida de mundo pequeńo, w. También puede observarse que el coeficiente medio de agrupamiento en ambas observaciones es muy alto $(C \approx .80)$ indicando que las unidades léxicas mencionadas para el centro de interés aprendizaje se encuentran altamente conectadas a unidades similares, ya sea semántica o fonológicamente.

Tabla 4. Descriptivos y métricas de los grafos

\begin{tabular}{|l|c|c|}
\hline & Observación 1 & Observación 2 \\
\hline Nodos (unidades léxicas) & 155 & 165 \\
\hline Aristas & 868 & 1558 \\
\hline Diámetro con pesos, diam $(G)$ & 9 & 8 \\
\hline Longitud media de camino, $l_{\mathrm{G}}$ & 1.927 & 1.885 \\
\hline Grado medio, $<k>$ & 11.200 & 18.885 \\
\hline Coeficiente medio de agrupamiento, $C$ & .807 & .818 \\
\hline Coeficiente de agrupamiento de grafo aleatorio, $C_{\text {rand }}$ & .072 & .114 \\
\hline Longitud media de camino de grafo aleatorio, $L_{\text {rand }}$ & 2.088 & 1.738 \\
\hline Coeficiente de mundo pequeño $\sigma$ & 12.098 & 6.587 \\
\hline Medida de mundo pequeño, $w$ & .091 & -.075 \\
\hline Exponente $\gamma$ de distribución de grado & 2.58 & 3.78 \\
\hline
\end{tabular}

Nota: el total de nodos considera al centro de interés aprendizaje. Se declaran los coeficientes de agrupamiento y longitud media de camino de grafo aleatorio utilizados para los coeficientes y medidas de mundo pequeño. 
Tabla 5. Unidades léxicas con mayor grado en dos medidas en grafos asociativos

\begin{tabular}{|c|l|c|l|c|}
\hline \multicolumn{1}{|c|}{ Unidad Léxica } & $k_{1}$ & \multicolumn{1}{c|}{ Unidad Léxica } & $k_{2}$ \\
\hline 1. & profesor & 78 & Conocimiento & 114 \\
\hline 2. & conocimiento & 74 & motivación & 96 \\
\hline 3. & enseńanza & 72 & constructivismo & 76 \\
\hline 4. & alumno & 65 & proceso & 71 \\
\hline 5. & proceso & 52 & profesor & 68 \\
\hline 6. & escuela & 43 & conducta & 64 \\
\hline 7. & memoria & 42 & metacognición & 63 \\
\hline 8. & educación & 33 & cambio / construcción(+) & 62 \\
\hline 9. & evaluación & 27 & aprendiz & 59 \\
\hline 10. & aprender / estudiar / libro & 26 & enseñanza / esquema $(+)$ & 56 \\
\hline 11. & desarrollo & 25 & Social & 52 \\
\hline 12. & comprensión & 24 & cognición / memoria & 51 \\
\hline 13. & inteligencia / leer(-) & 23 & experiencia & 48 \\
\hline 14. & aula & 22 & adquisición & 47 \\
\hline 15. & cambio & 21 & alumno & 46 \\
\hline
\end{tabular}

Nota: (-) Unidades léxicas que desaparecen; (+) Unidades léxicas que se incorporan.

A nivel de nodos (tabla 5) se aprecian valores más altos en el grado en la segunda observación, indicando aumento en la conectividad de las unidades léxicas. Aquellas unidades que presentan grado mayor son las que concentran un mayor número de aristas y por lo tanto son las más centrales o importantes.

Respecto de la distribución de grado de las unidades léxicas, los exponentes $\gamma$ de distribución de grado de las redes muestran que estas corresponden a una distribución con ley de potencia, siendo entonces redes libres de escala (Barabási y Albert 1999). Los valores críticos de la distribución con ley de potencia muestran valores característicos para esa distribución $(\gamma=2.58$, en la primera y $\gamma=3.78$, en la segunda observación).

\subsection{Análisis cualitativo de las redes léxicas}

El análisis cualitativo se hizo considerando las unidades léxicas más conectadas y sus relaciones, ya sea entre ellas y/o con otras unidades que muestran una menor conectividad pero que se relacionan con ellas, consideradas nodos vecinos. Este análisis permitió observar la presencia de núcleos de unidades léxicas altamente relacionadas que serían las más utilizadas para conceptualizar el aprendizaje, diferentes en las dos observaciones. 
En la primera observación, como se observa en la Figura 1, las unidades léxicas con mayor disponibilidad y conectividad son profesor, conocimiento, enseñanza, alumno y proceso, seguidas por escuela y memoria. Las cinco primeras se relacionan fuertemente entre ellas formando cliques (conjunto de 3 o más nodos, que se encuentran totalmente conectados entre sí) que muestran relaciones semánticas y de co-ocurrencia. Así, las relaciones más fuertes se dan entre los nodos profesor, alumno y enseñanza; luego conocimiento, enseñanza y proceso; y finalmente enseñanza, proceso y profesor.

Para esclarecer más los significados, se realizó un análisis de las unidades léxicas vecinas, el que mostró que profesor se relacionaba además con escuela y memoria; alumno con memoria y aula y conocimiento con saber, aprender y profesor. Estas relaciones sugieren que el concepto aprendizaje en la primera observación podría caracterizarse como un proceso determinado esencialmente por condicionantes externos, en donde el conocimiento es entregado por el profesor y reproducido por el alumno.

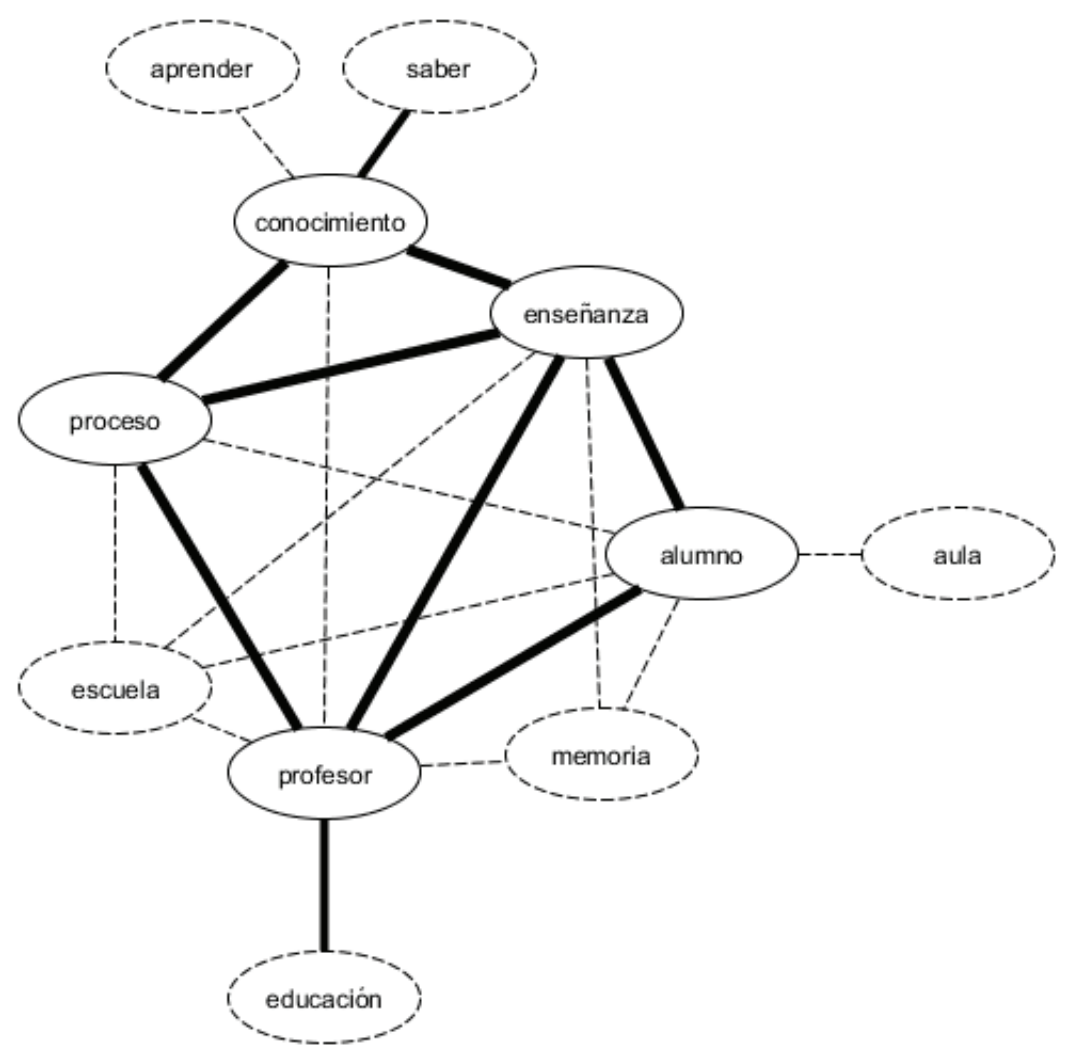

Figura 1: grafo del concepto aprendizaje con unidades léxicas principales y sus vecinas en la primera observación 
En la segunda observación (ver Figura 2), las relaciones se complejizan, apareciendo determinantes internos con mayor importancia. Las unidades léxicas con mayor disponibilidad y más conectadas son conocimiento, motivación, constructivismo y proceso; seguidas por profesor, conducta, metacognición, cambio y construcción. Todas estas unidades léxicas muestran fuertes relaciones entre sí, observándose mayor conectividad y disponibilidad en la unidad léxica conocimiento, desplazando a profesor que aparecía como más importante en la primera observación. Entre los cliques o triángulos de los que participa conocimiento, pueden destacarse aquellos que forma con metacognición y constructivismo; con cambio y construcción; proceso y cambio, y motivación y profesor.

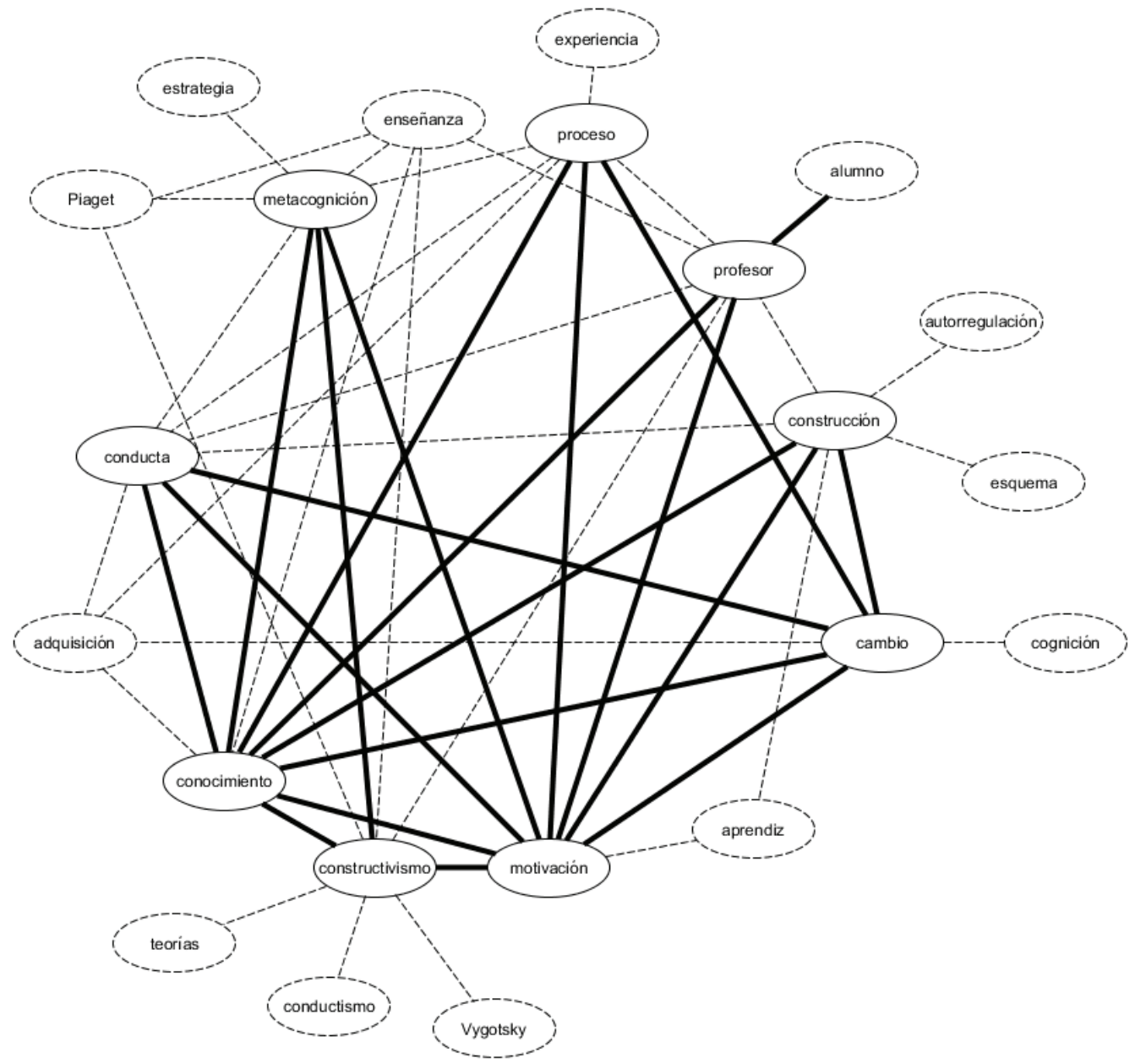

Figura 2: grafo del concepto aprendizaje con nodos principales y sus vecinos en la segunda observación 
La segunda unidad léxica más importante fue motivación, formando cliques con profesor y conocimiento; con metacognición y conocimiento; construcción y cambio; proceso y conocimiento, y profesor y conocimiento.

Otros cambios observados corresponden a la incorporación de construcción, que muestra como vecinos a autorregulación y la nueva unidad léxica esquema, además de formar un clique con motivación y aprendiz. Esta última unidad aumenta considerablemente su disponibilidad y conectividad, superando a alumno, la que junto con disminuir su conectividad muestra mayor relación con determinantes externos del aprendizaje. Por su parte, aprendiz muestra mayor relación con procesos internos como motivación, conocimiento y conducta.

Los cambios observados en las unidades léxicas más importantes y sus relaciones sugieren que en la segunda observación, los participantes se refieren al aprendizaje con unidades léxicas que consideran aspectos más procesuales y determinantes internos que en la primera. La presencia de procesos cognitivos más complejos (metacognición, construcción) y el aumento de importancia de motivación sugieren un desplazamiento hacia una concepción de aprendizaje referida a determinantes internos de tipo cognitivo y afectivo.

Resulta interesante también observar los cambios que experimentan las unidades léxicas más importantes de la primera observación: profesor, conocimiento, enseñanza, alumno y proceso. En la primera observación se observan fuertes conexiones entre sí y con unidades referidas a contextos y/o procesos superficiales (memoria) o poco definidos (aprender). En la segunda observación, en cambio, muestran relaciones más fuertes con palabras referidas a procesos internos, a la vez que la unidad léxica enseñanza pierde preeminencia relacionándose menos directamente con el resto de la red.

En sentido contrario, las unidades léxicas más conectadas en la segunda observación mostraban relaciones más fuertes con determinantes externos (enseñanza, profesor, escuela) y poco definidos (memoria, aprender) en la primera observación. Solo constructivismo mostraba relación con elementos teóricos coherentes desde una perspectiva piagetiana (Piaget, proceso, adaptación, desarrollo), aunque también relacionado con enseñanza.

Los resultados muestran también cambios en las epistemologías subyacentes a las concepciones de aprendizaje. A partir de los cambios en la red de vecinos de la unidad léxica conocimiento, si en la primera observación se encontraba fuertemente relacionada con enseñanza, proceso, saber y profesor; en la segunda, además de relacionarse con la unidad que le sigue en importancia (motivación) y con otras que muestran alta frecuencia (conducta y cambio), también lo hace con unidades que refieren a teorías y procesos constructivos (constructivismo, construcción), además de las unidades metacognición, adquisición y proceso. Puede decirse que la concepción de conocimiento comienza a desplazarse de ser "algo" que el profesor y la enseñanza disponen a un proceso más bien bajo las disposiciones del aprendiz, acercándose a posiciones relativistas. 


\section{DisCUSIÓN Y CONCLUSIONES}

En cuanto a la disponibilidad léxica, los resultados de este estudio muestran incremento en los totales y promedio de respuestas por participante y disminución del total de palabras diferentes, de manera similar a lo encontrado en estudiantes universitarios para el léxico matemático (Urzúa et al. 2006) y para estudiantes de $1^{\text {er }}$ y $2^{\circ}$ año de educación media (Ferreira et al. 2014; Salcedo y del Valle 2013), asimismo se observó un leve aumento en el índice de cohesión.

El incremento en los totales y promedio de respuestas por participante, indica que los estudiantes aumentaron su léxico latente entre las dos observaciones; sin embargo, este aumento se produce con un reemplazo importante de unidades léxicas. Casi el 50\% de las unidades es reemplazado por otras nuevas, manteniéndose un núcleo importante de unidades léxicas de uso corriente al hablar de aprendizaje, como son conocimiento, enseñanza, profesor, alumno o educación.

La disminución del total de unidades léxicas diferentes y el leve aumento del índice de cohesión sugieren que el léxico de los participantes se hace algo más homogéneo, disminuyendo las unidades únicas y aumentando las compartidas. Se ha planteado que la disponibilidad de las unidades léxicas depende de los fenómenos cognitivos de frecuencia y recencia, es decir del uso de estas y de su novedad (Ellis 2012), lo que podría explicar la desaparición, incorporación y mayor disponibilidad de algunas unidades emitidas. Sin embargo, al no disponer de estudios longitudinales similares, no es posible establecer comparaciones respecto de la evolución del léxico en grupos o personas.

$\mathrm{El}$ incremento de nodos entre las dos observaciones implica un aumento en el total de unidades léxicas compartidas por los participantes. Asimismo, se observa un aumento importante en la conectividad entre las unidades, la que casi se duplica. En cuanto a las características estructurales de los grafos léxicos, se observa que ambas redes poseen estructuras de mundo pequeño, presentando longitudes medias de camino más corto levemente inferiores a 2, cumpliendo tanto con lo propuesto para estructuras de mundo pequeño (Watts y Strogatz 1998), así como para las características propias de las redes léxicas (Borge-Holthoefer y Arenas 2010a).

Los indicadores $\gamma$ de distribución de grado señalan que ambas redes presentan ley de potencia coincidiendo con lo observado en redes léxicas construidas con grandes bases de datos (Borge-Holthoefer y Arenas 2010a; Steyvers y Tenenbaum 2005), indicando que las probabilidades de conexión de las nuevas unidades léxicas son mayores con aquellas unidades que presentaban mayor conectividad en la primera observación, según el principio de agregamiento preferencial (Barabási y Albert 1999).

En cuanto a las concepciones de aprendizaje, los análisis, tanto cuantitativos como cualitativos de los nodos muestran cambios en las unidades léxicas utilizadas para conceptualizar el aprendizaje. En la primera observación, las unidades más conectadas se referían tanto a determinantes externos o contextuales del aprendizaje (enseñanza, profesor, educación), como a procesos poco definidos, altamente dependientes de contexto para su significación 
(conocimiento, memoria, aprender), sugiriendo proximidad con una teoría implícita directa (Pozo et al. 2006) y con una concepción reproductiva del aprendizaje. Esto coincide con lo descrito en diversas investigaciones desde una perspectiva fenomenográfica, ya sea como un enfoque superficial del aprendizaje en estudiantes suecos (Marton y Säljö 2005); o como concepciones del aprendizaje en estudiantes finlandeses (Paakkari et al. 2011; Tynjälä 1997); australianos (Purdie et al. 1996), y españoles y portugueses (Rosário et al. 2006).

En la segunda observación, las unidades léxicas más importantes refieren principalmente a elementos procesuales y determinantes internos que formaron parte de los contenidos del curso, a la vez que permanecen unidades importantes en la primera observación, aunque con diferencias respecto de su conectividad. Es así como la unidad léxica conocimiento aumenta su importancia, pasando a ocupar el primer lugar, mientras que profesor desciende al quinto puesto y enseñanza al décimo. Las unidades que incrementan su conectividad sugieren el paso hacia una concepción de aprendizaje más bien cualitativo, que integra elementos de una teoría implícita interpretativa con elementos constructivos, próxima a los perfiles mixtos de teorías implícitas y concepciones de aprendizaje encontrados en poblaciones similares, tanto en Chile (Cárcamo y Castro 2015; Gómez et al. 2010; Judikis et al. 2008) como en otras realidades geográficas (Marton y Säljö 2005; Rosário et al. 2006; Tynjälä 1997; García y Vilanova 2008).

La persistencia de determinantes externos, de relaciones no del todo coherentes en la segunda observación (por ejemplo, conocimiento-conducta), y la apariencia de listado de títulos de contenidos tratados en el curso sugieren que, más que una restructuración fuerte (Carey 1986), se ha producido una reestructuración débil o asimilación en el sentido piagetiano. Una posible explicación puede encontrarse en la Teoría del concepto umbral (Meyer y Land 2003), que considerando aspectos semánticos en el cambio conceptual, plantea que la reestructuración de los significados de conceptos centrales en una disciplina, como puede ser el aprendizaje en la formación de futuros profesores, implica una fase liminal para el aprendiz que enfrentado a un cambio epistemológico y ontológico utiliza nuevos conceptos, sin tener completa claridad de su significado (Land, Rattray y Vivian 2014).

Finalmente, es posible también que por efecto de recencia (Ellis 2012), los nuevos conceptos aparezcan incorporados en el léxico de los participantes con una mayor conectividad y frecuencia. Esto es similar a lo observado por Tynjälä (1997) al implementar una intervención dirigida al desarrollo de una concepción constructivista del aprendizaje en estudiantes finlandeses. Por otra parte, el análisis de grafos léxicos ha mostrado que ciertas relaciones de co-ocurrencia son más robustas debido a su mayor frecuencia de uso (BorgeHolthoefer y Arenas 2010b; Ferrer i Cancho y Solé 2001), como pueden ser en este caso las asociaciones enseñanza-aprendizaje y profesor-alumno, unidades léxicas que además forman parte de un léxico nuclear para referirse a los procesos de enseñanza y aprendizaje.

Los resultados de este estudio muestran que el léxico referido al aprendizaje de los participantes experimenta cambios luego de un semestre académico, utilizando una metodología no tradicional que puede aportar al conocimiento tanto de las concepciones de aprendizaje, como al estudio de redes léxicas. 
Respecto de las concepciones de aprendizaje los análisis cuantitativos y cualitativos muestran similitudes con hallazgos realizados en estudios fenomenográficos de concepciones de aprendizaje y de teorías implícitas de aprendizaje a través de cuestionarios de dilemas, esta vez a partir de los significados compartidos por una comunidad de hablantes.

En cuanto al análisis de redes o grafos léxicos, los resultados aquí presentados, aun tratándose de una muestra pequeña, coinciden con los de redes construidas con grandes bases de datos. Resulta interesante que estas redes más pequeñas presenten las mismas características topológicas y puedan ser también caracterizadas como redes complejas, corroborando las formas de crecimiento observadas en otras investigaciones. Por último, a diferencia de otros estudios que analizan disponibilidad léxica y/o utilizan metodología de grafos, se trata de un estudio longitudinal que permite conocer la evolución de significados.

Finalmente, las limitaciones de este estudio sugieren que sus resultados deben ser tomados con cautela. Por una parte, la utilización del análisis de grafos para redes léxicas tiene un carácter más bien exploratorio en el estudio de conceptos específicos. Por tratarse de un diseño pre-experimental sin grupo control, no puede asegurarse con certeza que los cambios fueron producto sólo de la participación y metodología del curso. Sin embargo, tanto las nuevas unidades léxicas, como aquellas que aumentaron su disponibilidad y conectividad, muestran una alta coincidencia con los contenidos del curso, sugiriendo que éste tuvo relación con este cambio.

La metodología de grafos, unida a la disponibilidad léxica, permite observar y analizar diversos aspectos del lenguaje, desde las unidades básicas como las unidades léxicas, hasta llegar al lenguaje en sí en tanto estructura dinámica, y su observación en estudios longitudinales permite analizar la forma en que estos se organizan y reestructuran a partir de procesos de aprendizaje. Así, la metodología de grafos es una metodología que resultaría pertinente para observar procesos como los cambios en la estructura de un concepto, cambio conceptual o reestructuración del aprendizaje.

Esta metodología puede ser enriquecida en conjunto con otras, ya sea cualitativas o cuantitativas, así como con la introducción de otras variables y el diseño de intervenciones en poblaciones distintas a la de este estudio.

\section{ANEXo: Fórmulas Para LaS MÉTRICAS DE LOS GRAFOS}

\begin{tabular}{|l|l|}
\hline \multicolumn{1}{|c|}{ Métrica } & \multicolumn{1}{c|}{ Fórmula } \\
\hline Grado $k$ & Suma de todas las aristas que se conectan a un nodo. \\
\hline Grado medio $<k>$ & $<k>=\frac{1}{n} \sum_{i} k_{i}$ \\
& donde $k_{i}$ es el grado correspondiente al grado $i$ \\
\hline
\end{tabular}




\begin{tabular}{|c|c|}
\hline $\begin{array}{l}\text { Diámetro, } D \text {, para un } \\
\text { grafo con pesos }\end{array}$ & $\begin{array}{l}\operatorname{diam}(G):=\max \{\operatorname{dist} G, w(u, v) \mid u \in V(G), v \in V(G)\} \text { donde } \operatorname{diam}(G) \\
\text { es el diámetro del grafo } G ; \max \{\operatorname{dist} G, w(u, v)\} \text { es la máxima distancia } \\
\text { entre los nodos } u, v \text { del grafo ponderado } G, w \text { y } V \text { es el conjunto finito } \\
\text { de nodos (Havet 2010). }\end{array}$ \\
\hline $\begin{array}{l}\text { Longitud media de } \\
\text { camino más corto, } l_{\mathrm{G}} \\
\text { para un grafo no dirigido }\end{array}$ & $\begin{array}{l}l_{G}=\frac{2}{n \times(n-1)} \times \sum_{i \neq j} d\left(v_{i}, v_{j}\right) \\
\text { donde } n \text { es el número de nodos del grafo } G\end{array}$ \\
\hline $\begin{array}{l}\text { Distribución de grado } \\
P(k) \text { : }\end{array}$ & $\begin{array}{l}p(k)=n_{k} / n \\
\text { donde } n k \text { señala el grado de un nodo escogido al azar y } n \text { el máximo de } \\
\text { aristas que puede tener el grafo. }\end{array}$ \\
\hline $\begin{array}{l}\text { Coeficiente medio de } \\
\text { agrupamiento } C\end{array}$ & $\begin{array}{l}\text { Para calcular el coeficiente medio de agrupamiento es necesario calcular } \\
\text { previamente el coeficiente de agrupamiento local para cada nodo } i, C i \\
\text { con la fórmula: } \\
C_{i}=\frac{2 T_{i}}{k_{i}\left(k_{i}-1\right)} \\
\text { donde } T_{i} \text { es el número de aristas entre los nodos vecinos del nodo } i \text { y } k_{i} \\
\text { es el grado del nodo. El coeficiente medio de agrupamiento del grafo, } C \\
\text { se calcula promediando todos los } C_{i} \text {. Si } N \text { es el total de nodos, entonces: } \\
C=\sum_{i=1}^{N} \frac{C_{i}}{N}\end{array}$ \\
\hline $\begin{array}{l}\text { Coeficiente de mundo } \\
\text { pequeńo, } \sigma\end{array}$ & 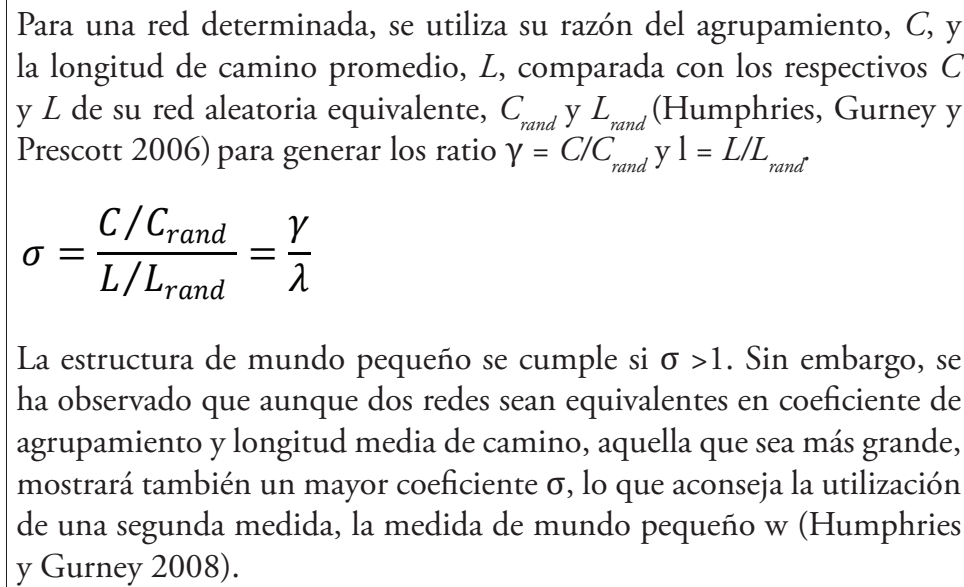 \\
\hline
\end{tabular}




\begin{tabular}{|c|c|}
\hline $\begin{array}{l}\text { Medida de mundo } \\
\text { pequeño, } w\end{array}$ & $\begin{array}{l}\omega=\frac{L_{\text {rand }}}{L}-\frac{C}{C_{\text {latt }}} \\
\text { Para determinar el coeficiente de mundo pequeño w, se comparan el } \\
\text { coeficiente de agrupamiento, } C \text {, de la red con su equivalente de lattice, } \\
C_{\text {latt }} \text { y la longitud media de camino de una red aleatoria equivalente, } \\
L_{\text {rand }} \text { con la longitud media de camino de la red, } L \text { (Telesford et al. } \\
2011) \text {. } \\
\text { La medida de mundo pequeño w presenta valores entre }-1 \text { y } 1 \text {, sin } \\
\text { importar el tamaño de la red. Los valores cercanos a } 0 \text { indican mundo } \\
\text { pequeño. Valores positivos señalan características más cercanas a un } \\
\text { grafo aleatorio: } L \approx L_{\text {rand }} \text { y } C<<C_{\text {latt }} \text {. Valores negativos indican un grafo } \\
\text { más regular: } L>>L_{\text {rand }} \text { y } C \approx C_{\text {latt }} \text { (Telesford et al., 2011). }\end{array}$ \\
\hline
\end{tabular}

\section{Obras citadas}

Alba, Orlando. 2013. "Variación diacrónica del léxico disponible dominicano". Lingüística española actual 35.1: 149-180.

Albert, Réka y Albert-László Barabási. 2002. "Statistical mechanics of complex networks." Reviews of Modern Physics 74.1: 47-96.

Alexandridis, Kostas y Yiheyis Maru. 2012. "Collapse and reorganization patterns of social knowledge representation in evolving semantic networks." Information Sciences 200: 1-21.

Ávila-Muñoz, Antonio. 2016. "El léxico disponible y la enseñanza del español. Propuesta de selección léxica basada en la teoría de los conjuntos difusos”. Journal of Spanish Language Teaching 3.1: 31-43.

. 2017. "The available lexicon: A tool for selecting appropriate vocabulary to teach a foreign language". Iranian Journal of Language Teaching Research 5.1: 71-91.

Ávila-Muñoz, Antonio y José María Sánchez-Sáez. 2014. "Fuzzy sets and Prototype Theory Representational model of cognitive community structures based on lexical availability trials". Review of Cognitive Linguistics 12.1: 133-159.

Barabási, Albert-László y Réka Albert. 1999. "Emergence of scaling in random networks". Science 286.5439: 509-512.

Bermúdez Valle, Álvaro, Guillermo Bustillo Romero y Alex Escobar Blanco. 2016. “Disponibilidad léxica en el campo de la política de estudiantes de segundo año de bachillerato a nivel nacional”. Anuario de Investigación 2013. Universidad Evangélica de El Salvador: 45-88.

Blas, José Luis y Manuela Casanova. 2001-2003. “Interacción de factores sociales y de adscripción lingüística en el léxico disponible de una comunidad escolar española”. 
Lenguas Modernas 28-29: 165-189.

Borge-Holthoefer, Javier y Alex Arenas. 2010a. "Categorizing words through semantic memory navigation". The European Physical Journal B 74: 265-270. . 2010b. "Semantic networks: structure and dynamics". Entropy 12: 1264-1302.

Borge-Holthoefer, Javier, Yamir Moreno y Alex Arenas. 2012. "Topological versus dynamical robustness in a lexical network". International Journal of Bifurcation and Chaos 22.7: 1250157-1-1250157-9.

Campbell, Donald y Julian Stanley. 1995. Diseños experimentales y cuasiexperimentales en la investigación social. Buenos Aires: Amorrortu.

Cárcamo, Rodrigo y Pablo Castro. 2015. "Concepciones sobre el aprendizaje de estudiantes de pedagogía de la Universidad de Magallanes y docentes en ejercicio en la educación básica de la ciudad de Punta Arenas, Chile”. Formación Universitaria 8.5: 13-24.

Carey, Susan. 1986. "Cognitive science and science education". American Psychologist 40.10: 1123-1130.

Castorina, José A. y Ricardo J. Baquero. 2005. Dialéctica y psicología del desarrollo. Buenos Aires: Amorrortú.

Cepeda, Milko, Ángela Cárdenas, Macarena Carrasco, Nicole Castillo, Joselyne Flores, Constanza González y Melanie Oróstica. 2016. "Relación entre disponibilidad léxica y comprensión lectora, en un contexto de educación técnico profesional rural". Sophia Austral 18: 81-93.

Cepeda, Milko, Maribel Granada y María Pomes. 2014. "Disponibilidad léxica en estudiantes de primero básico”. Literatura y Lingüística 30: 181-206.

Cerda, Gamal, Pedro Salcedo, Carlos Pérez y Verónica Marín. 2017. "Futuros Profesores de Matemáticas: Rol de la Disponibilidad Léxica, Esquemas de Razonamiento Formal en Logros Académicos Durante su Formación Inicial”. Formación universitaria 10.1: 33-46.

Collins, Allan y Elizabeth Loftus. 1975. "A spreading-activation theory of semantic processing”. Psychological Review 82.6: 407-428.

Colombo, Natalia, Olga Trevisán y Hugo Wingeyer. "2013. Aportes para las representaciones sobre la sexualidad en la disponibilidad léxica de estudiantes secundarios de la región NEA". De Prácticas y discursos. Cuadernos de Ciencias Sociales 2.2. Disponible en http://depracticasydiscursos.unne.edu.ar/Revista2/pdf/

Schaufler.pdf

del Valle, María, Pedro Salcedo y Anita Ferreira. 2016. "Analyzing the Availability of Lexicon in Mathematics Education Using no Traditional Technological Resources". International Journal of Supply Chain Management 5.2: 144-149.

Dogorovtsev, Sergey y José F. Mendes. 2001. "Language as an evolving word web”. Proceedings of the Royal Society of London. Series B: Biological Sciences 268.1485: 2603-2606.

Echeverría, Max, et al. 1987. "Disponibilidad léxica en educación media”. RLA. Revista de Lingüística Teórica y Aplicada 25: 55-115. 
Echeverría, Max, et al. 2008. "DispoGrafo: una nueva herramienta computacional para el análisis de relaciones semánticas en el léxico disponible”. RLA. Revista de Lingüística Teórica y Aplicada 46.1: 81-91.

Ellis, Nick C. 2012. "What can we count in language, and what counts in language acquisition, cognition, and use?" Frequency effects in language learning and processing. Ed. Stefan Th, De Gries and Dagmar Divjak. Berlin/Boston: De Gruyter Mouton: 7-34.

Elman, Jeffrey. 2004. "An alternative view of the mental lexicon". Trends in Cognitive Sciences 8.7: 301-306.

Fasce, Eduardo, et al. 2009. "Atributos del profesionalismo estimados por estudiantes de medicina y médicos. Análisis mediante el modelo de disponibilidad léxica”. Revista Médica de Chile 137.6: 746-752.

Fernández-Merino, Pablo. 2011. "Disponibilidad léxica de inmigrantes: propuesta para una necesidad”. Lengua y migración 3.2: 83-105.

Ferreira, Anita, Pedro Salcedo y María del Valle. 2014. "Estudio de disponibilidad léxica en el ámbito de las matemáticas". Estudios filológicos 54: 69-84.

Ferreira, Roberto y Max Echeverría. 2010. "Redes semánticas en el léxico disponible de inglés L1 e inglés LE”. Onomázein: Revista de Lingüística, Filología y Traducción de la Pontificia Universidad Católica de Chile 21: 133-153.

Ferrer i Cancho, Ramón y Ricard Solé. 2001. "The small world of human language". Proceedings of the Royal Society of London. Series B: Biological Sciences 268.1482: 2261-2265.

García, María y Silvia Vilanova. 2008. "Las representaciones sobre el aprendizaje de los alumnos de profesorado. Diseño y validación de instrumento para analizar concepciones implícitas sobre el aprendizaje en profesores de matemática en formación". Revista Electrónica de Investigación en Ciencias 3.2: 27-34.

Garzón Segura, Anni y Luis Alberto Penagos López. 2016. "Disponibilidad léxica en estudiantes de primer semestre de pregrado de una institución universitaria de Villavicencio, Colombia”. Forma y Función 29.2: 63.48.

Gephi.org. 2014. The Open Graph Viz Platform. Disponible en https:/gephi.github.io/.

Gómez, Viviana y Paula Guerra. 2012. "Teorías implícitas respecto a la enseñanza y el aprendizaje: ¡existen diferencias entre profesores en ejercicio y estudiantes de pedagogía?” Estudios Pedagógicos 38.1: 25-43.

Gómez, Viviana, Paula Guerra y María Paz González. 2010. "Explorando el cambio epistemológico y conceptual en la formación inicial de profesores en distintos contextos universitarios". Evidencias para políticas públicas en Educación. Santiago de Chile: Ministerio de Educación: 45-82.

González, María Isabel. 2003. "Anglicismos innecesarios en el habla culta de Las Palmas de Gran Canaria”. Epos: Revista de filología 19: 193-218

Hargreaves, Andy. 2010. "Presentism, Individualism, and Conservatism: the legacy of Dan Lortie's Schoolteacher: a Sociological Study". Curriculum Inquiry 40.1: 143-154.

Harispe, Sébastien, et al. 6 December 2013. "Semantic Measures for the Comparison of 
units of language, concepts or instances from text and knowledge base analysis". arxiv.org. Disponible en https://arxiv.org/ftp/arxiv/papers/1310/1310.1285.pdf>.

Havet, Frédéric. 2010. "Algorithms in edge-weighted graphs". Combinatorial Optimization-Algorithms for telecommunications. Disponible en http://www-sop.inria.fr/ members/Frederic.Havet/

Henríquez Guarín, María Clara, Viviana Mahecha Mahecha y Geral Eduardo Mateus Ferro. 2016. "Análisis de los mecanismos cognitivos del léxico disponible de cuerpo bumano a través de grafos" Lingüística y Literatura 69: 229-251.

Hernández-Muñoz, Natividad. 2015. "La evaluación de la competencia léxica adulta: una aproximación a través de la disponibilidad léxica y la especialización académica en preuniversitarios”. Revista de Filología 33:79-99.

Hernández-Muñoz, Natividad, Cristina Izura y Andrew W. Ellis. 2006. "Cognitive aspects of lexical availability”. European Journal of Cognitive Psychology 18.5: 730-755.

Humphries, Mark D. y Kevin Gurney. 2008. "Network 'small-world-ness': a quantitative method for determining canonical network equivalence". PLoS One 3.4: e0002051.

Humphries, Mark D., Kevin Gurney y Tony J. Prescott. 2006. "The brainstem reticular formation is a small-world, not scale-free, network". Proceedings of the Royal Society B: Biological Sciences 273.1585: 503-511.

Jiménez Catalán, R.M., and J. Ojeda Alba. 2009a. Disponibilidad léxica en inglés como lengua extranjera en dos tipos de instrucción. Lenguaje y Textos 30: 166-176.

Jiménez Catalán, R.M., and J. Ojeda Alba. 2009b. Girls' and boys' lexical availability in EFL. ITL International Journal of Applied Linguistics 158: 57-76.

Judikis, Juan Carlos et al. 2008. “Teorías implícitas sobre el aprendizaje y su relación con las prácticas pedagógicas en estudiantes de pedagogía de la Universidad de Magallanes y profesores en ejercicio de la XII Región. “ Informe de final de Proyecto FONIDE (Fondo de Investigación y Desarrollo de la Educación). Universidad de Magallanes.

Karelovic, Bruno. 2010. Estudio de la estructura de grafos de disponibilidad léxica. Memoria para optar al título de Ingeniero Civil Matemático. Departamento de Ingeniería Matemática. Concepción: Universidad de Concepción.

Land, Ray, Julie Rattray y Peter Vivian. 20014. "Learning in the liminal space: a semiotic approach to threshold concepts”. Higher Education 67: 199-217.

López, Humberto. 1996. "Los estudios de disponibilidad léxica: pasado y presente". Boletín de Filología 35.1: 245-259.

Luján, Carmen Isabel y Alicia Bolaños. 2014. "Disponibilidad léxica y anglicismos informáticos en los centros de interés: internet, software, hardware”. Odisea 15: 101-126.

Martínez-Fernández, Reinaldo. 2007. "Concepción de aprendizaje y estrategias metacognitivas en estudiantes universitarios de psicología”. Anales de Psicología 23.1: 7-16.

Marton, Ference y Roger Säljö. 2005. "Approaches to learning”. The Experience of Learning: Implications for teaching and studying in higher education. Ed. Ference Marton, Dai Hounsell and Noel Entwistle. $3^{\mathrm{a}}$ ed. Edinburgh: University of Edinburgh, Centre for Teaching, Learning and Assessment. 39-58. 
Meyer, Jan H. F. y Ray Land. 2003. "Threshold concepts and troublesome knowledge: linkages to ways of thinking and practising within the disciplines". Improving student learning - 10 years on. Ofxord: OCSLD. 412-424.

Morais, Ana Sofia, Henrik Olsson y Lael J. Schooler. 2013. "Mapping the structure of semantic memory”. Cognitive Science 37.1: 125-145.

Motter, Adilson, et al. 2002. "Topology of the conceptual network of language". Physical Review E 65.6: 065102.

Murillo, Marielos. 1999. "Crecimiento de la disponibilidad léxica: niños de preescolar y primer ciclo de la educación básica costarricense”. Filosofía y Lingüística 25.2: 187203.

Neuman, Yair. 2006. "A theory of meaning". Information Sciences 176.10: 1435-1449.

Newman, Mark E.J., Steven Strogatz y Duncan Watts. 2001. "Random graphs with arbitrary degree distributions and their applications". Physical Review E 64.2: 026118.

Pacheco, Antonio, Selina Ponce Castañeda, Salvador A. Palomares Sánchez. 2016. "Disponibilidad Léxica Matemática en estudiantes de Ingeniería y Ciencias”. UNIÓN, Revista Iberoamericana de Educación Matemática 47: 44-61.

Paakkari, Leena, Paivi Tynjälä y Lasse Kannas. 2011. "Critical aspects of student teachers' conceptions of learning”. Learning and Instruction 21.6: 705-714.

Paredes, Florentino. 2012. "Desarrollos teóricos y metodológicos recientes de los estudios de disponibilidad léxica”. Revista Nebrija de Lingüística Aplicada. Disponible en http://www.nebrija.com/revista-linguistica/desarrollos-teoricos-y-metodologicosrecientes-de-los-estudios-de-disponibilidad-lexica.

Peñaranda, María, et al. 2006. "Los "Small Worlds" y el algoritmo de Floyd: una manera de estudiar la colaboración científica”. Psicothema 18.1: 78-83.

Peñaranda-Ortega, María, Elena Quiñones-Vidal y Julia Osca-Lluch. 2009. "La revista Anales de Psicología desde una perspectiva de redes sociales". Anales de Psicología 25.2: 199-208.

Pérez Durán, Marco Antonio. 2015. "Estudio de los campos semánticos que sirven en la construcción de la unidad fraseológica del tipo peyorativo". Forma y Función 28.1: 157-182.

Pintrich, Paul R. 2006. "Las creencias motivacionales como recursos y restricciones para el cambio conceptual”. Cambio conceptual y educación. Ed. Wolfgang Schnotz, Stella Vosniadou and Mario Carretero. Buenos Aires: Aique. 53-86.

Pozo, Juan Ignacio. 1998. Aprendices y maestros. Madrid: Alianza Editorial,. 1993. "Psicología y Didáctica de las ciencias de la naturaleza ¿concepciones alternativas?” Infancia y Aprendizaje 16.62-63: 187-204.

Pozo, Juan Ignacio, et al. 2006. "Las teorías implícitas sobre el aprendizaje y la enseñanza”. Ed. Pozo, Juan I., et al. Nuevas formas de pensar la enseñanza y el aprendizaje: Las concepciones de profesores y alumnos. Barcelona: Graó. 95-132.

Purdie, Nola, John Hattie y Graham Douglas. 1996. "Student conceptions of learning and their use of self-regulated learning: Strategies: a cross-cultural comparison”. Journal 
of Educational Psychology 88.1: 87-11.

Rodríguez, Lizeth, Juan López Chávez y María Rebeca Padilla. 2014. "Léxico disponible entre jóvenes y adultos sobre tecnologías de la información y comunicación y ciberlenguaje". Investigium IRE: Ciencias Sociales y Humanas 5.1: 113-128.

Rodríguez, Francisco e Isabel Muñoz. 2011. "Disponibilidad léxica sobre palabras específicas en estudiantes de educación secundaria de Almería”. Espiral. Cuadernos del profesorado 4.8: 22-31.

Rosário, Pedro, et al. 2006. "Perspectiva fenomenográfica de las concepciones del aprendizaje". Revista Galego-Portuguesa de Psicoloxía de la Educación 13.11-12: 195-206.

Rosas, Ricardo y Christian Sebastián. 2008. Piaget, Vigotski y Maturana. Constructivismo a tres voces. Buenos Aires: Aique.

Salcedo, Pedro y María del Valle. 2013. "Disponibilidad léxica matemática en estudiantes de enseñanza media de Concepción, Chile". Atenas 4.21: 1-16.

Salcedo, Pedro, et al. 2014. "Plataforma adaptativa online para el fortalecimiento de las competencias matemáticas y pedagógicas a partir del estudio léxico semántico de estudiantes y profesores de Pedagogía en Matemática”.

Sánchez, Lourdes. 2005. "Concepciones de aprendizaje de profesores universitarios y profesionales no docentes: un estudio comparativo". Anales de Psicología 21 .2: 231-243.

Sánchez, Víctor y Marielos Murillo. 1993. "Campos semánticos y disponibilidad léxica en preescolares”. Revista Educación 17.2: 15-25.

Santos, Saúl. 2015. "Disponibilidad léxica en náayeri (cora) y español de estudiantes bilingues de primaria: un estudio contrastivo". Antropología. Boletín Oficial del INAH 99: 37-49.

Saussure, Ferdinand de. 2007. Curso de lingüística general. Madrid: Losada.

Solé, Ricard, et al. 2010. "Language networks: their structure, function and evolution". Complexity 15.6: 20-26.

Steyvers, Mark y Joshua B. Tenenbaum. 2005. "The large-scale structure of semantic networks: statistical analyses and a model of semantic growth". Cognitive Science 29.1: 41-78.

Studer, Rudi, V. Richard Benjamins y Dieter Fensel. 1998. "Knowledge engineering: principles and methods". Data \& Knowledge Engineering 25.1: 161-197.

Telesford, Qawi K., et al. 2011. "The ubiquity of small-world networks". Brain Connectivity $1.5: 367-375$.

Tomé, Carmela. 2016. "Vocabulario de la informática y las nuevas tecnologías. Caracterización desde la disponibilidad léxica”. Caracteres. Estudios culturales y críticos de la esfera digital 5.1: 112-139.

Tynjälä, Päivi. 1997. 'Developing education students' conceptions of the learning process in different learning environments". Learning and Instruction 7.3: 277-292.

Urzúa, Paula, Katia Sáez y Max Echeverría. 2006. “Disponibilidad léxica matemática. Análisis cuantitativo y cualitativo". RLA. Revista de Lingüística Teórica y Aplicada 44.2: 59-76. 
Valencia, Alba. 1997. "Disponibilidad léxica. Muestreo y estadísticos”. Onomázein: Revista de Lingüística, Filología y Traducción de la Pontificia Universidad Católica de Chile 2: $197-226$.

Vitevich, Michael. 2008. "What can graph theory tell us about word learning and lexical retrieval?” Journal of Speech, Language, and Hearing Research 51.2: 408-422.

Watts, Duncan J. y Steven H. Strogatz. 1998. "Collective dynamics of "small-world" networks". Nature 393.6684: 440-442.

Windschitl, Mark. 2002. "Framing Constructivism in practice as the negotiation of dilemmas: an analysis of the conceptual, pedagogical, cultural, and political challenges facing teachers". Review of Educational Research 72.2: 131-175.

Wingeyer, Hugo. 2014a. "Temas de la disponibilidad léxica de la región NEA de argentina: mitos populares, la ciudad, el campo, insultos y diversiones nocturnas. Aportes para la elaboración de programaciones interculturales y de materiales en la enseñanza de E/LE”. Signos ELE 8: 1-14.

. 2014b. "Disponibilidad léxica de la región NEA de Argentina. Análisis SocioSemiótico de formas asociadas al acto de insultar". Contextos 31: 129-141

Wittgenstein, Ludwig. 1999. Investigaciones filosóficas. Barcelona: Altaya.

Zortea, Maxciel, et al. 2014. "Graph analysis of semantic word association among children, adults and the elderly”. Psicologia: Reflexão e Crítica 27.1: 90-99. 
